\title{
Independent associations of physical activity and cardiorespiratory fitness with metabolic risk factors in children: the European youth heart study
}

\author{
U. Ekelund $\cdot$ S. A. Anderssen $\cdot$ K. Froberg • \\ L. B. Sardinha $\cdot$ L. B. Andersen $\cdot$ S. Brage $\cdot$ \\ European Youth Heart Study Group
}

Received: 8 May 2007 / Accepted: 5 June 2007 / Published online: 20 July 2007

(C) Springer-Verlag 2007

\begin{abstract}
Aims/hypothesis High levels of cardiorespiratory fitness (CRF) and physical activity (PA) are associated with a favourable metabolic risk profile. However, there has been no thorough exploration of the independent contributions of cardiorespiratory fitness and subcomponents of activity (total PA, time spent sedentary, and time spent in light, moderate and vigorous intensity PA) to metabolic risk factors in children and the relative importance of these factors.

Methods We performed a population-based, cross-sectional study in 9- to 10- and 15- to 16-year-old boys and girls from three regions of Europe $(n=1709)$. We examined the independent associations of subcomponents of PA and CRF with metabolic risk factors (waist circumference, BP, fasting
\end{abstract}

U. Ekelund $(\bowtie) \cdot$ S. Brage

MRC Epidemiology Unit, Elsie Widdowson Laboratory, Fulbourn Road,

Cambridge CB1 9NL, UK

e-mail: Ulf.ekelund@mrc-epid.cam.ac.uk

S. A. Anderssen · L. B. Andersen

Department of Sports Medicine,

Norwegian University of Sport Sciences,

Oslo, Norway

K. Froberg

Institute of Sport Science and Clinical Biomechanics,

University of Southern Denmark,

Odense, Denmark

L. B. Sardinha

Faculty of Human Movement Sciences,

Technical University of Lisbon,

Lisbon, Portugal glucose, insulin, triacylglycerol and HDL-cholesterol levels). Clustered metabolic risk was expressed as a continuously distributed score calculated as the average of the standardised values of the six subcomponents.

Results CRF (standardised $\beta=-0.09,95 \% \mathrm{CI}-0.12,-0.06$ ), total PA (standardised $\beta=-0.08,95 \% \mathrm{CI}-0.10,-0.05$ ) and all other subcomponents of PA were significantly associated with clustered metabolic risk. After excluding waist circumference from the summary score and further adjustment for waist circumference as a confounding factor, the magnitude of the association between CRF and clustered metabolic risk was attenuated (standardised $\beta=-0.05,95 \%$ CI -0.08 , $-0.02)$, whereas the association with total PA was unchanged (standardised $\beta=-0.0895 \% \mathrm{CI}-0.10,-0.05$ ).

Conclusions/interpretation PA and CRF are separately and independently associated with individual and clustered metabolic risk factors in children. The association between $\mathrm{CRF}$ and clustered risk is partly mediated or confounded by adiposity, whereas the association between activity and clustered risk is independent of adiposity. Our results suggest that fitness and activity affect metabolic risk through different pathways.

Keywords Aerobic fitness $\cdot$ Exercise $\cdot$ Metabolic syndrome

$\begin{array}{ll}\text { Abbreviations } \\ \text { CRF } & \text { cardiorespiratory fitness } \\ \text { CVD } & \text { cardiovascular disease } \\ \text { EYHS } & \text { European Youth Heart Study } \\ \text { ICC } & \text { intraclass correlation coefficient } \\ \text { LPA } & \text { light intensity physical activity } \\ \text { MPA } & \text { moderate intensity physical activity } \\ \text { PA } & \text { physical activity } \\ \text { VPA } & \text { vigorous intensity physical activity }\end{array}$




\section{Introduction}

Moderate to high levels of cardiorespiratory fitness (CRF) and physical activity (PA) have been consistently associated with lower risks of many health outcomes, such as cardiovascular disease (CVD) [1-4], the metabolic syndrome [5-7], type 2 diabetes [8-10] and all-cause mortality $[11,12]$ in adults. These associations are also sometimes independent of fatness [10], although the relative importance of fatness, activity and CRF remains controversial [13].

PA and CRF may also be associated with CVD and metabolic risk factors in children. However, most studies have addressed the association between either CRF or PA with a single risk factor and few have addressed the joint contribution of CRF and activity to these risk factors. The most convincing studies have suggested an association between activity with insulin sensitivity $[14,15]$ or between CRF and insulin sensitivity $[15,16]$. However, it is unclear whether these associations are independent of adiposity [16-19].

Total body movement (i.e. the total amount of PA) and patterns of activity (i.e. time spent sedentary and in activities of varying intensity levels, and bouts of sustained activity) are separate dimensions of activity that may be associated with individual metabolic risk factors and clustered metabolic risk in different ways. However, these subdimensions of activity are inherently difficult to measure precisely in epidemiological studies. We have recently reported that the objectively measured total amount of PA, assessed by accelerometry, is associated with clustered metabolic risk in a large population-based sample of European children [20], and that this association is independent of the amount of time spent viewing television and other confounding factors [21]. We have also reported that CRF is associated with clustered metabolic risk [22]. However, none of our previous studies examined the independent associations between subdimensions of PA, CRF and adiposity with metabolic risk factors. Moreover, the relative importance of PA and CRF for these risk factors in children was not explored in our previous reports. This information is important as it may inform primary preventive action in children.

Therefore, the aim of the present study was to examine the independent associations of subdimensions of PA and CRF with individual metabolic risk factors and with clustered metabolic risk in a large population-based sample of children from three distinct geographical locations in Europe.

\section{Methods}

Study design The European Youth Heart Study (EYHS) is a mixed longitudinal study designed to examine the nature and strength of personal, environmental and lifestyle influences on CVD risk factors in European children and the interactions among these influences. The rationale, aims, study design, selection criteria and sample size are described in detail elsewhere [23].

The present cross-sectional study included 1,092 children aged 9-10 years (544 boys and 548 girls) and 829 adolescents aged 15-16 years (367 boys and 462 girls) from three geographically defined areas in Europe (the city of Odense, Denmark, the city and surrounding rural areas of Tartu, Estonia, and the island of Madeira, Portugal). Data on anthropometric variables, sexual maturity and biochemical data were available for all children $(n=1921)$. Of these children, $1,709(89 \%)$ had valid data on objectively measured PA, aerobic fitness and the risk factors included in the composite score, and constitute the sample for the present report. Written informed consent was obtained from a parent or guardian and the study procedures were explained verbally to all children. Ethical approval for the study was obtained from the local research ethics committees in each study region.

Measurements Weight and height were measured using standard techniques with the participants in light clothing and without shoes. BMI was calculated as weight $(\mathrm{kg}) /$ height $(\mathrm{m})^{2}$. Four skinfold thickness measurements (triceps, biceps, subscapula and suprailiac; $\mathrm{mm}$ ) were taken on the left side of the body in duplicate or triplicate, according to the criteria described by Lohman et al. [24], and the two closest measurements at each site were averaged. Fat mass and fat-free mass were calculated using age- and sexspecific equations [25].

Resting systolic and diastolic BPs were measured in the sitting position, after 5 min of sitting rest, with a Dinamap vital signs monitor (Critikron, Tampa, FL, USA). Five measurements were taken at 2 min intervals and the means of the last three measurements were averaged and used in analysis.

Sexual maturity was assessed by the data collectors, using the five-stage scale for breast development in girls and pubic hair in boys, according to Tanner [26]. Smoking status (no smoking, regular smoking) was obtained by selfreport. Children's birthweight and parental socioeconomic status (highest education and income) were obtained by self-report from the parents.

PA Free-living PA was assessed with an MTI Actigraph (Manufacturing Technology, Fort Walton Beach, FL, USA) accelerometer over two weekdays and two weekend days, as previously described $[20,21]$. The outcome variables were daily activity (cpm), time (min) spent sedentary $(<500 \mathrm{cpm})$, time spent in light intensity PA (LPA; 5012,000 cpm), time spent in moderate intensity PA (MPA; 
2,001-3,999 cpm) and time spent in vigorous intensity PA (VPA; $>4,000 \mathrm{cpm}$ ). Daily cpm is an indicator of the total amount of PA (i.e. the average intensity of PA). This variable was derived by dividing total counts by monitoring time per day and averaging over the measurement period. This variable is significantly correlated with PA energy expenditure obtained by the doubly labelled water method [27]. Time spent sedentary and in LPA, MPA and VPA were expressed as percentages of total monitored time to account for differences between individuals in time wearing the accelerometer. We excluded all time blocks with ten or more consecutive zero counts, assuming that the monitor was not worn.

$C R F$ CRF was assessed during an incremental ergometer cycle test to exhaustion on an electronically braked ergometer and expressed as watts per kilogram fat-free mass per min (W $\mathrm{kg} \mathrm{FFM}^{-1} \mathrm{~min}^{-1}$ ). In an independent subsample of 9- and 15-year-old children the test-retest CV was $2.5-4.8 \%$ and was highly correlated with measured $V \mathrm{O}_{2 \max }(r=0.90 ; p<0.001 ; \mathrm{S}$. A. Anderssen and L. B. Andersen, unpublished results).

Initial and incremental workloads were $25 \mathrm{~W}$ for 9-yearolds weighing $<30 \mathrm{~kg}$ and $30 \mathrm{~W}$ for heavier children. For 15 -year-old boys and girls the initial workloads were 40 and $50 \mathrm{~W}$ respectively. Workloads were increased every third minute until exhaustion. Heart rate was continuously measured every $5 \mathrm{~s}$ throughout the test (Polar Vantage; Polar Electro Oy, Kempele, Finland). Criteria for achieving a maximal test was a heart rate $>185$ beats per min and a subjective judgement that the child could not continue even after verbal encouragement.

Biochemistry Overnight fasting blood samples were taken in the morning from the antecubital vein. Samples were divided into aliquots, separated within $30 \mathrm{~min}$ and stored at $-80^{\circ} \mathrm{C}$ until transport to WHO-certified laboratories for analyses. Samples from Denmark and Estonia were measured in one laboratory (Bristol, UK), whereas samples from Portugal were measured separately in a second laboratory (Cambridge, UK), as previously described [21]. Briefly, HDL-cholesterol and triacylglycerol were measured by enzymatic methods in all samples (Olympus Diagnostica, Hamburg, Germany). Glucose was analysed using the hexokinase method, measured with an Olympus AU600 autoanalyser (Olympus Diagnostica) in all samples. Insulin was analysed using enzyme immunoassay (microtitre plate format; Dako Diagnostics, Ely, UK) in the Bristol laboratory and by two-site immunometric assays with either ${ }^{125} \mathrm{I}$ or alkaline phosphatase labels in the Cambridge laboratory. Between-laboratory correlations for 30 randomly selected samples analysed at both laboratories were 0.94-0.98. However, before analyses, all biochemical data were standardised to the mean ( $z$ score) by study location, sex and age group.

Clustered metabolic risk score We constructed a standardised, continuously distributed variable (standardised to the mean by sex, age group and study location) for clustered metabolic risk, which we have described in detail previously $[7,21,28]$. This variable was derived by standardising and then summing the following continuously distributed indices of adiposity (waist circumference) to create a $z$ score: hypertension ([systolic BP+diastolic BP]/2); hyperglycaemia (fasting plasma glucose); insulin resistance (fasting insulin); fasting HDL-cholesterol $\times-1$; and fasting triacylglycerol $z$ score. We also calculated a risk score without the adiposity component (i.e. waist circumference) to examine whether the associations between the main exposures (PA and $\mathrm{CRF}$ ) and clustered metabolic risk were affected by including adiposity in the risk score. The purpose of using a continuously distributed variable was to maximise statistical power [29].

Statistics Descriptive data are presented as mean $\pm \mathrm{SD}$ and geometric mean and $95 \%$ CI. Fasting insulin, sum of four skinfold thicknesses and time spent in VPA were logarithmically transformed (log) owing to their skewed distributions. Differences between sexes, study locations and age groups were tested by ANOVA. Associations between variables were analysed by simple correlation coefficients and partial correlations after adjustment for age, sex and study location. Multiple regression analyses were used to analyse the independent associations between PA variables (total counts, time spent sedentary, and in LPA, MPA and VPA) and CRF with individual risk factors. Interaction factors (i.e. age $\times$ main exposures and sex $\times$ main exposures) were considered to evaluate whether age and sex modified the associations between PA variables and CRF with risk factors. Our primary model was adjusted for sex, age and study location. We then assessed the independent associations between PA variables and CRF with each individual risk factor after further adjustment for adiposity (i.e. waist circumference). In our final model we also adjusted our analysis for birthweight, sexual maturity, smoking status, maternal BMI and parental socioeconomic status in addition to all other confounding factors.

We then tested whether subdimensions of PA and CRF were independently associated with the clustered risk score in two models. The first model (adiposity-dependent) included all individual risk factors and was adjusted for the confounders described above. In the second model (adiposity-independent), the adiposity component was excluded from the outcome, and additionally adjusted for. This analysis examined whether adiposity could act as a mediator and/or confounder. All data were analysed in their 
continuous form, although data are stratified by quartiles of PA and CRF for illustrative purposes. Data were analysed with SPSS for Windows (version 11.0) and $p<0.05$ denoted statistical significance.

\section{Results}

Table 1 shows the descriptive characteristics of the participants. Total PA was significantly greater in younger than in older children $(684 \pm 255$ vs $530 \pm 209 \mathrm{cpm}, p<0.0001)$ and in boys than girls $(689 \pm 285$ vs $555 \pm 200 \mathrm{cpm}, p<0.0001)$. CRF was significantly higher in older than younger children $(3.95 \pm 0.73$ vs $3.47 \pm 0.72, p<0.0001)$ and in boys than girls $(3.85 \pm 0.72$ vs $3.51 \pm 0.76, p<0.0001)$. A significant sex $\times$ age interaction was observed for CRF ( $p=0.045)$, indicating that the influence of age was somewhat greater in boys than girls.

Table 2 shows the partial correlations of subcomponents of PA with CRF and risk factors. All PA estimates were weakly but significantly correlated with most of the risk factors, except HDL-cholesterol. Similarly, PA subcomponents were weakly and significantly correlated with CRF $(r=0.08$ to $0.14, p<0.0001)$ after adjustment for sex, age and study location. Most subcomponents of PA were highly and significantly correlated with each other $(r=0.13$ to $0.89, p<0.0001)$. CRF was significantly and inversely correlated with waist circumference $(r=-0.20, p<0.0001)$, sum of skinfold thicknesses $(r=-0.18, p<0.0001)$, fasting glucose $(r=-0.15, p<0.0001)$ and insulin $(r=-0.07$, $p<0.0001$ ), but significantly and positively correlated with diastolic BP $(r=0.10, p<0.0001)$, systolic BP $(r=0.11$, $p<0.0001)$ and triacylglycerol $(r=0.07, p=0.005)$.

Table 3 shows the independent associations between subdimensions of PA, CRF and the metabolic risk factors. All models are adjusted for age, sex and study location. Additionally, CRF is included in all models with the subcomponents of PA, and all models are additionally adjusted for waist circumference except when waist circumference is the outcome. No significant interactions of sex or age with PA or CRF were observed, except when systolic BP was modelled as the outcome variable (age $\times \mathrm{PA}, p$ for interaction=0.023). Total PA and percentage of time spent sedentary and in LPA, MPA and VPA were significantly associated with most of the metabolic risk factors, except for waist circumference and HDL-cholesterol after adjustment for CRF. The magnitude of association was stronger for the total amount of activity and percentage time spent

Table 1 Descriptive characteristics of participants

\begin{tabular}{|c|c|c|c|c|c|}
\hline & \multicolumn{2}{|l|}{9 years } & \multicolumn{2}{|l|}{15 years } & \\
\hline & $\begin{array}{l}\text { Boys } \\
(n=504)\end{array}$ & $\begin{array}{l}\text { Girls } \\
(n=504)\end{array}$ & $\begin{array}{l}\text { Boys } \\
(n=334)\end{array}$ & $\begin{array}{l}\text { Girls } \\
(n=404)\end{array}$ & \\
\hline Age (years) & $9.7 \pm 0.4$ & $9.6 \pm 0.4$ & $15.5 \pm 0.5$ & $15.5 \pm 0.5$ & $\mathrm{G}^{* * *}, \mathrm{~S}^{* *}$ \\
\hline Weight (kg) & $33.3 \pm 7.0$ & $32.9 \pm 7.1$ & $62.4 \pm 10.4$ & $55.8 \pm 8.4$ & $\mathrm{G}^{* * *}, \mathrm{~S}^{* * *}, \mathrm{G} \times \mathrm{S}^{* * *}$ \\
\hline Height (cm) & $137.8 \pm 6.4$ & $137.7 \pm 6.6$ & $172.8 \pm 7.8$ & $163.7 \pm 6.5$ & $\mathrm{G}^{* * *}, \mathrm{~S}^{* * *}, \mathrm{G} \times \mathrm{S} * * *$ \\
\hline BMI & $17.4 \pm 2.7$ & $17.2 \pm 2.7$ & $20.8 \pm 2.8$ & $20.8 \pm 2.7$ & $\mathrm{G}^{* * *}$ \\
\hline Waist (cm) & $60.3 \pm 6.5$ & $58.0 \pm 6.4$ & $72.1 \pm 6.5$ & $67.1 \pm 5.7$ & $\mathrm{G}^{* * *}, \mathrm{~S}^{* * *}, \mathrm{G} \times \mathrm{S}^{* * *}$ \\
\hline Skinfold thickness (mm) & $28.6(26.9,30.2)$ & $34.9(34.1,35.6)$ & $30.8(29.9,31.8)$ & $45.8(44.4,46.8)$ & $\mathrm{G}^{* * *}, \mathrm{~S}^{* * *}, \mathrm{G} \times \mathrm{S}^{* * *}$ \\
\hline Diastolic BP (mmHg) & $58.9 \pm 7.6$ & $59.7 \pm 6.7$ & $61.6 \pm 6.9$ & $63.1 \pm 6.4$ & $\mathrm{G}^{* * *}, \mathrm{~S}^{* *}$ \\
\hline Systolic BP (mmHg) & $101.5 \pm 9.7$ & $101.0 \pm 9.2$ & $114.4 \pm 12.3$ & $106.9 \pm 9.0$ & $\mathrm{G}^{* * *}, \mathrm{~S}^{* * *}, \mathrm{G} \times \mathrm{S}^{* * *}$ \\
\hline Glucose (mmol/1) & $5.17 \pm 0.35$ & $5.07 \pm 0.39$ & $5.27 \pm 0.45$ & $5.10 \pm 0.40$ & $\mathrm{G}^{* * *}, \mathrm{~S} * * *, \mathrm{G} \times \mathrm{S} *$ \\
\hline HDL-cholesterol (mmol/l) & $1.52 \pm 0.30$ & $1.47 \pm 0.28$ & $1.30 \pm 0.27$ & $1.40 \pm 0.27$ & $\mathrm{G}^{* * *}, \mathrm{~S}^{*}, \mathrm{G} \times \mathrm{S} * * *$ \\
\hline Triacylglycerol (mmol/l) & $0.69 \pm 0.31$ & $0.80 \pm 0.34$ & $0.83 \pm 0.49$ & $0.89 \pm 0.39$ & $\mathrm{G}^{* * *}, \mathrm{~S} * * *$ \\
\hline Insulin $(\mathrm{pmol} / \mathrm{l})$ & $5.35(5.18,5.52)$ & $6.64(6.50,6.78)$ & $9.27(8.95,9.60)$ & $10.87(10.52,11.22)$ & $\mathrm{G}^{* * *}, \mathrm{~S}^{* * *}$ \\
\hline Total activity (cpm) & $750 \pm 280$ & $616 \pm 205$ & $594 \pm 236$ & $478 \pm 165$ & $\mathrm{G}^{* * *}, \mathrm{~S}^{* * *}$ \\
\hline Time sedentary $(\%)$ & $62.2 \pm 10.8$ & $66.3 \pm 9.0$ & $71.5 \pm 9.7$ & $75.8 \pm 7.5$ & $\mathrm{G}^{* * *}, \mathrm{~S}^{* * *}$ \\
\hline Time in LPA (\%) & $26.3 \pm 6.5$ & $25.5 \pm 5.2$ & $19.6 \pm 6.6$ & $17.7 \pm 5.8$ & $\mathrm{G}^{* * *}, \mathrm{~S}^{* * *}$ \\
\hline Time in MPA $(\%)$ & $8.9 \pm 4.4$ & $6.6 \pm 3.2$ & $6.3 \pm 3.4$ & $4.8 \pm 2.5$ & $\mathrm{G}^{* * *}, \mathrm{~S}^{* * *}, \mathrm{G} \times \mathrm{S} * *$ \\
\hline Time in VPA $(\%)$ & $2.5 \pm 2.1$ & $1.6 \pm 1.3$ & $2.6 \pm 2.4$ & $1.6 \pm 1.5$ & $\mathrm{~S} * * *$ \\
\hline $\mathrm{CRF}\left(\mathrm{W} \mathrm{kg} \mathrm{FFM}{ }^{-1} \min ^{-1}\right.$ ) & $3.62 \pm 0.68$ & $3.32 \pm 0.73$ & $4.20 \pm 0.65$ & $3.76 \pm 0.74$ & $\mathrm{G}^{* * *}, \mathrm{~S} * * *, \mathrm{G} \times \mathrm{S}^{*}$ \\
\hline
\end{tabular}

Data for insulin and skinfold thickness are geometric mean and $95 \% \mathrm{CI}$; other data are mean \pm SD $(n=1709)$

$F F M$, fat-free mass; $G$, age group; $S$, sex

$* p<0.05$

$* * p<0.01$

$* * * p<0.001$ 
sedentary compared with time spent in LPA, MPA and VPA. Similarly, all subcomponents of PA were also significantly and inversely associated with the summary score of metabolic risk, the greatest magnitude of association being observed for total PA (standardised $\beta=-0.08$, $95 \%$ CI $-0.10,-0.05)$. When excluding waist circumference from the summary score and additionally adjusting for waist circumference as a confounding factor, the magnitude of association was unchanged. CRF was significantly and inversely associated with waist circumference (standardised $\beta=-0.11,95 \%$ CI $-0.17,-0.5$ ), fasting glucose (standardised $\beta=-0.09,95 \% \mathrm{CI}-0.16,-0.06)$, HDL-cholesterol $\times 10^{-1}$ (standardised $\beta=-0.07,95 \%$ CI $-0.12,-0.01$ ), insulin (standardised $\beta=-0.13,95 \%$ CI $-0.19,-0.08$ ) and the metabolic summary score with waist circumference (standardised $\beta=-0.09,95 \% \mathrm{CI}-0.12,-0.06$ ) and without waist circumference (standardised $\beta=-0.05,95 \% \mathrm{CI}-0.8$, -0.02 ), after adjustment for age, sex, country and total PA.

Figure 1 shows the associations between total PA and CRF stratified by quartiles with clustered metabolic risk. A highly significant inverse association was observed for the association between $\mathrm{CRF}$ and the metabolic risk score ( $p$ for trend $<0.0001$ ) and between PA and clustered metabolic risk ( $p$ for trend $<0.0001$ ).

Additional adjustments for birthweight, sexual maturity, smoking status, maternal BMI and parental socioeconomic status did not change the direction or magnitude of associations of CRF and subcomponents of PA with individual and clustered metabolic risk (data not shown). In this model, total PA was significantly and independently associated with five (systolic and diastolic BP, fasting glucose, fasting triacylglycerol and fasting insulin) out of the seven individual risk factors and CRF was significantly and independently associated with four of the risk factors.

We then replaced waist circumference with sum of skinfold thicknesses (log-transformed) and reanalysed our data; the associations were unchanged (data not shown). No significant interactions between PA and CRF were observed in any of our analyses (data not shown).

\section{Discussion}

Our results suggest that PA and CRF are independently associated with indicators of insulin resistance, hyperglycaemia, hyperlipidaemia and clustered metabolic risk in children. These associations are independent of body fatness and other potential confounding factors. Our observations may have public health implications because increasing the total amount of PA may have beneficial effects regardless of the level of CRF and body fatness in children.

Limitations of our study include its cross-sectional design, which limits inferences about causality and its direction.
Although we controlled for several confounding factors, such as age, sex, study location, birthweight, socioeconomic status and sexual maturity, we cannot exclude the possibility that other, unmeasured confounding factors, such as genetic variation, energy intake and patterns, and other socio-cultural factors, could explain our observations. Only randomisation within a trial can deal with issues of unmeasured confounding.

Our main exposure variables were probably measured with different degrees of error. CRF is fairly stable over time (i.e. several months) and reasonably precisely measured within an individual. In contrast, PA is highly variable within children, and 3 or 4 days of accelerometry measurement may not capture a fully representative snapshot of this complex behaviour [30]. Thus, our estimates of the magnitude of association between PA and metabolic outcomes may be underestimated. The within-individual intraclass correlation coefficient (ICC, inter-individual variance/total variance) for the total amount of PA measured by accelerometry has been estimated to 0.5 [30]. Assuming all measurement error stems from within-individual variability in PA, the ICC can be used to adjust the observed regression coefficients for measurement error by dividing the regression coefficient by the ICC [31]. This may then mean that the true magnitude of associations between PA and metabolic outcomes may be twice as strong as those reported here.

CRF was normalised by fat-free mass to account for between-individual differences in body size [32]. This may be a potential source of bias since fat-free mass is correlated with waist circumference (partial $r=0.55$; data not shown) and with the sum of skinfold thicknesses (partial $r=0.17$; data not shown) even after adjustment for age and sex in our cohort. Therefore, some residual confounding from adiposity may persist in our models and the observed magnitude of associations between CRF and metabolic risk factors may be slightly overestimated. Indeed, when excluding waist circumference from the outcome variable (i.e. clustered metabolic risk score) and adjusting for it as a confounding factor, the magnitude of association between $\mathrm{CRF}$ and clustered risk was attenuated and weaker than that between PA and clustered metabolic risk. An alternative explanation may be that waist circumference is part of the causal pathway between CRF and clustered metabolic risk. If this is the case, adjusting for waist circumference as a confounding variable will diminish a true association between CRF and clustered risk.

We included all children who achieved a heart rate of at least 185 beats per min when terminating the exercise test. This may not be a maximal effort for all children and the estimated mean values for CRF may be slightly underestimated. However, it is unlikely that this will affect the observed associations between CRF, PA and risk factors. Indeed, our results were unchanged after further adjustment for maximal heart rate (data not shown). 


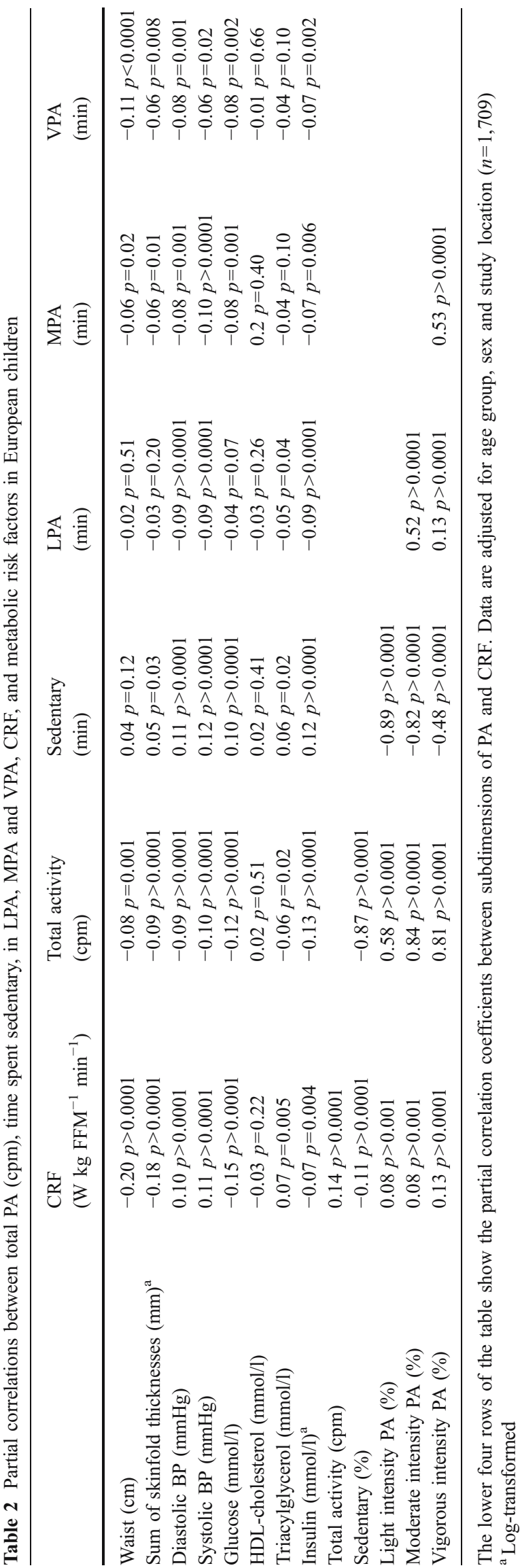

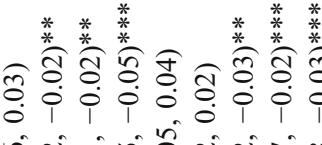

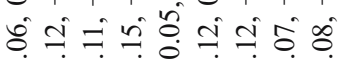
i i i i i i i i

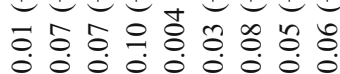

i $\dot{i}^{\circ}$ i

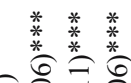

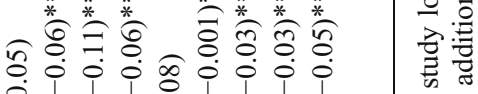

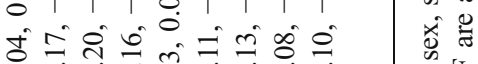

a i i i o o io

i i i i i i I I

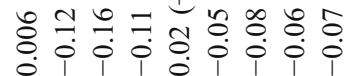

递

苟 密

氜 흉

范

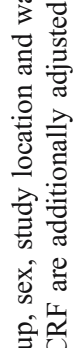

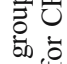

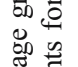

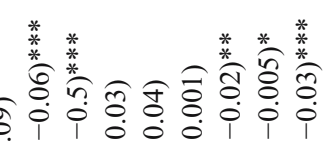

苞造

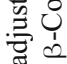

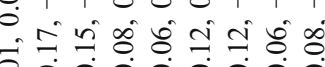

0. 0 i 0 i i

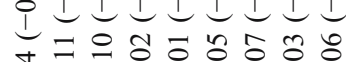

可

ڤี

总

-

ชิ

으ำำ 으응

\& 0000000

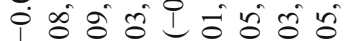

mé丶

i

证

蹗

焉

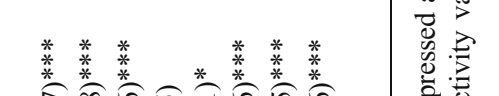

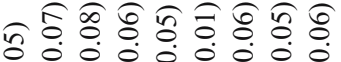

- $\begin{aligned} & 0 \\ & 0\end{aligned}$ i 00 个

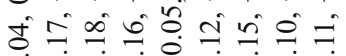

i i i i i i i o

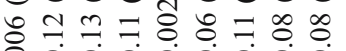

ठ웅

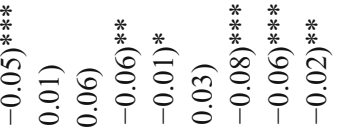

过

के

है 응

亏

चี ठठ :

Oิ

$\exists=$ है

产 :

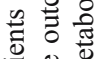

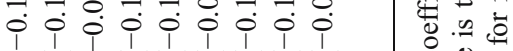

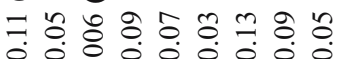

0 造

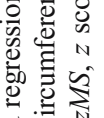

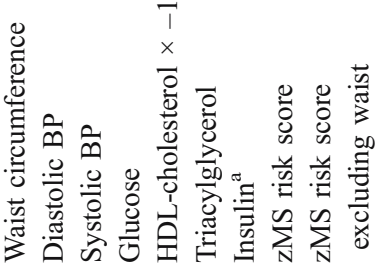

类

$\overrightarrow{8}$
$\stackrel{0}{0}$
$v$
v
*

$\ddot{z} \cdot \vec{a}$

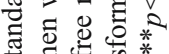

के 壳

要 苟

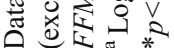




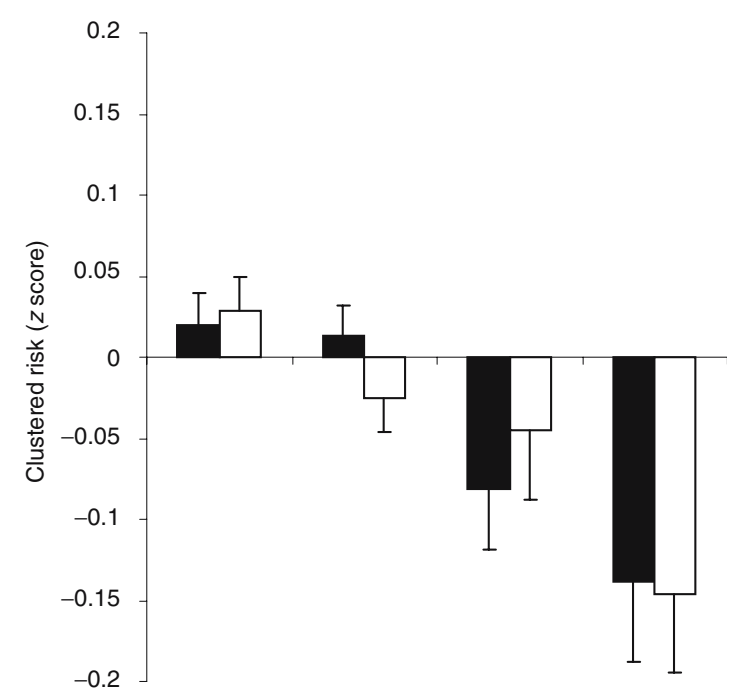

Fig. 1 Adjusted means $(95 \% \mathrm{CI})$ of clustered metabolic risk excluding adiposity in the risk score stratified by quartiles of aerobic fitness ( $p$ for trend $=0.06$ ) and total amount of PA ( $p$ for trend $<0.0001)$. Closed bars, aerobic fitness; open bars, PA. Data are adjusted for sex, age, study location, maximal heart rate and waist circumference $(n=1709)$

This is the first report from the EYHS examining the independent associations of different subcomponents of PA and CRF with metabolic risk in the entire EYHS cohort. In previous reports, we have observed an association between PA and insulin sensitivity in 9- to 10-year-old Danish children [14]. We have also reported that PA is inversely related to body fat in 9- to 10-year-old children [33], that $\mathrm{PA}$ is inversely associated with clustered metabolic risk [20], and that PA and television viewing are independently associated with clustered metabolic risk in both 9- to 10year-old and 15- to 16-year-old children [21]. We have also shown that aerobic fitness is inversely associated with clustered metabolic risk in both age groups [22]. Finally, in one of our previous reports, restricted to 9- to 10-year-old Danish children, we observed that aerobic fitness modified the association between overall PA and clustered metabolic risk [34]. In the present study, which comprised a much larger and diverse cohort of children, we were not able to replicate this finding. This may be explained by differences in sample size and the more homogeneous sample included in the previous report.

Strengths of our study include our large populationbased sample, our validated and objective method for measuring PA [27], the collection of fasting blood samples in a large, heterogeneous group of children, and the use of maximal incremental exercise testing to assess CRF.

Others have shown that $\mathrm{CRF}$ is associated with individual metabolic risk factors in youth [16-18, 35, 36]. However, these associations may be mediated at least partly through adiposity [17, 18]. Boreham et al. [35] suggested that the strength of association between fatness and CVD risk factors is greater than that between CRF and CVD risk factors in a large sample of Irish children. Shaibi et al. [37] reported that $\mathrm{CRF}$ was not related to any individual component of the metabolic syndrome independently of adiposity in overweight Hispanic children. Imperatore et al. [16] reported that both CRF and PA were significantly associated with insulin sensitivity independent of obesity in US adolescent boys but not in girls. Differences between studies may be attributable to different sample sizes, characteristics of the cohorts and differences in the methods employed. Our findings in a large population-based sample of European children demonstrate that both CRF and PA are associated with multiple individual risk factors and with clustered metabolic risk independently of adiposity.

CRF is partly influenced by genetic factors [38] and by regular exercise. CRF was weakly associated with PA in our study and the correlation between variables was similar to that reported by Imperatore et al. [16]. The strongest correlation between $\mathrm{CRF}$ and $\mathrm{PA}$ variables were between $\mathrm{CRF}$ and total body movement $(r=0.14 ; p<0.0001)$. Our results may then suggest that CRF and PA influence metabolic risk factors through separate pathways, or that $\mathrm{CRF}$ is a marker for specific muscle characteristics (e.g. muscle fibre type composition) that favour metabolic health. Indeed, an increased proportion of slow-twitch muscle fibres is associated with increased lipid oxidation [39].

In our more detailed analysis of the association between subcomponents of PA and metabolic risk factors, we observed that the percentage of time spent sedentary was significantly and positively associated with systolic and diastolic BP, fasting glucose, triacylglycerol and insulin, and with clustered metabolic risk. Time spent in MPA was also significantly but inversely associated with the same risk factors and the magnitudes of the associations were similar. Because of multicollinearity, we were unable to include the subcomponents of activity in the same model. However, these more detailed analyses of patterns of PA are important as they suggest that reducing the amount of time children spend sedentary and increasing the total amount of PA may have beneficial effects on metabolic and cardiovascular health.

Furthermore, the finding that PA is inversely associated with metabolic and CVD risk factors independently of CRF and adiposity may also have several important implications for public health. Increasing overall PA in children through play, active transport, leisure time PA and participation in sports is likely to have beneficial effects on children's metabolic risk profile. An association between PA, independently of CRF, is important because it may be more feasible to encourage children to participate in PAs, which do not necessarily have to be vigorous and associated with great levels of exhaustion, aiming to improve CRF. Although adiposity is a stronger predictor than PA of metabolic risk in children [21], PA may confer health 
benefits regardless of the degree of adiposity. Finally, our results were not modified by sex, age or study location, which suggest that these results are generalisable to a large proportion of European children.

CRF and PA are separately and independently associated with metabolic risk factors in children. The association between CRF and clustered risk is partly mediated or confounded by adiposity, whereas the association between PA and clustered risk is independent of adiposity. Our results may suggest that $\mathrm{CRF}$ and PA affect metabolic risk through different pathways.

Acknowledgements The authors are very grateful to the participants and their families who gave their time to the study. We would also like to acknowledge all the members of the European Youth Heart Study Group. This study was supported by grants from the following agencies: The Danish Heart Foundation, The Danish Medical Research Council Health Foundation, The Danish Council for Sports Research, The Foundation in Memory of Asta Florida Bolding Renée Andersen, The Faculty of Health Sciences, University of Southern Denmark, The Estonian Science Foundation (grant numbers 3277 and 5209) and The Medical Research Council, UK. The sponsors had no role in study design, data collection and analysis, the decision to publish, or preparation of the manuscript.

Duality of interest The authors declare that there is no duality of interest associated with this manuscript.

\section{References}

1. Blair SN, Kohl HW III, Paffenbarger RS Jr, Clark DG, Cooper KH, Gibbons LW (1989) Physical fitness and all-cause mortality: a prospective study of healthy men and women. JAMA 262:23952401

2. Lakka TA, Venalainen JM, Rauramaa R, Salonen R, Tuomiletho J, Salonen JT (1994) Relation of leisure-time physical activity and cardiorespiratory fitness to the risk of acute myocardial infarction. New Engl J Med 330:1549-1554

3. Carnethon MR, Gidding SS, Nehgme R, Sidney S, Jacobs DR Jr, Liu K (2003) Cardiorespiratory fitness in young adulthood and the development of cardiovascular disease risk factors. JAMA 290:3092-3100

4. Carnethon MR, Gulati M, Greenland P (2005) Prevalence and cardiovascular disease correlates of low cardiorespiratory fitness in adolescents and adults. JAMA 294:2981-2988

5. LaMonte MJ, Barlow CE, Jurca R, Kampert JB, Church TS, Blair SN (2005) Cardiorespiratory fitness is inversely associated with the incidence of metabolic syndrome: a prospective study of men and women. Circulation 112:505-512

6. Laaksonen DE, Lakka HM, Salonen JT, Niskanen LK, Rauramaa R, Lakka TA (2002) Low levels of leisure-time physical activity and cardiorespiratory fitness predict development of the metabolic syndrome. Diabetes Care 25:1612-1618

7. Ekelund U, Brage S, Franks PW, Emms S, Hennings S, Wareham NJ (2005) Physical activity energy expenditure predicts progression toward the metabolic syndrome independently of aerobic fitness in middle-aged healthy Caucasians: the Medical Research Council Ely Study. Diabetes Care 28:1195-1200
8. Lynch J, Helmrish SP, Lakka TA et al (1996) Moderately intense physical activities and high levels of cardiorespiratory fitness reduce the risk of non-insulin-dependent diabetes mellitus in middle-aged men. Arch Intern Med 156:1307-1314

9. Hu FB, Leitzmann M, Stampfer MJ, Colditz G, Willett W, Rimm E (2001) Physical activity and television watching in relation to risk for type 2 diabetes in men. Arch Intern Med 161:1542-1548

10. Bassuk SS, Manson JE (2005) Epidemiological evidence for the role of physical activity in reducing risk of type 2 diabetes and cardiovascular disease. J Appl Physiol 99:1193-1204

11. Pfaffenbarger RS Jr, Hyde RT, Wing AL, Hsieh CC (1986) Physical activity, all-cause mortality and longevity of college alumni. New Engl J Med 314:605-613

12. Laukkanen JA, Lakka TA, Rauramaa R et al (2001) Cardiovascular fitness as a predictor of mortality in men. Arch Intern Med 161:825-831

13. Blair SN, Church TS (2004) The fitness, obesity, health equation. Is physical activity the common denominator? JAMA 292:1232-1234

14. Brage S, Wedderkopp N, Ekelund U et al (2004) Objectively measured physical activity correlates with indices of insulin resistance in Danish children. The European Youth Heart Study (EYHS). Int J Obes 2004(28):1503-1508

15. Ku CY, Gower BA, Hunter GR, Goran MI (2000) Racial differences in insulin secretion and sensitivity in prepubertal children: role of physical fitness and physical activity. Obes Res 8:506-515

16. Imperatore G, Cheng YJ, Williams DE, Fulton J, Gregg EW (2006) Physical activity, cardiovascular fitness, and insulin sensitivity among U.S. adolescents: the National Health and Nutrition Examination Survey, 1999-2002. Diabetes Care 29:1567-1572

17. Lee SJ, Bacha F, Gungor N, Arslanian SA (2006) Cardiorespiratory fitness in youth: relationship to insulin sensitivity and $\beta$-cell function. Obesity 14:1579-1585

18. Ball GDC, Shaibi GQ, Cruz ML, Watkins MP, Weigensberg MJ, Goran MI (2004) Insulin sensitivity, cardiorespiratory fitness, and physical activity in overweight Hispanic youth. Obes Res 12:77-85

19. Platat C, Wagner A, Klumpp T, Schweitzer B, Simon C (2006) Relationships of physical activity with metabolic syndrome features and low-grade inflammation in adolescents. Diabetologia 49:2078-2085

20. Andersen LB, Harro M, Sardinha LB et al (2006) Physical activity and clustered cardiovascular risk in children: a cross-sectional study (The European Youth Heart Study). Lancet 368:299-304

21. Ekelund U, Brage S, Froberg K et al (2006) TV viewing and physical activity are independently associated with metabolic risk in children: the European Youth Heart Study. PLoS Med:e488

22. Anderssen SA, Cooper A, Riddoch C et al (2007) Low cardiorespiratory fitness is a strong predictor for clustering of cardiovascular disease risk factors in children independent of country, age and sex. Eur J Cardiovasc Prev Rehabil (in press)

23. Riddoch C, Edwards D, Page A et al (2005) The European Youth Heart Study-Cardiovascular Disease Risk Factors in Children: rationale, aims, study design, and validation of methods. J Phys Act Health 2:115-129

24. Lohman TG, Roche AF, Martorell R (eds) (1991) Anthropometric standardization reference manual. Human Kinetics Books, Champaign, IL

25. Slaughter MH, Lohman TG, Boileau RA et al (1988) Skinfold equations for estimation of body fatness in children and youth. Hum Biol 60:709-723

26. Tanner JM (1962) Growth at adolescence. Blackwell, Oxford

27. Ekelund U, Sjöström M, Yngve A et al (2001) Physical activity assessed by activity monitor and doubly labeled water in children. Med Sci Sports Exerc 33:275-281

28. Franks PW, Ekelund U, Brage S, Wong MY, Wareham NJ (2004) Does the association of habitual physical activity with the metabolic syndrome differ by level of cardiorespiratory fitness? Diab Care 27:1187-1193 
29. Ragland DR (1992) Dichotomizing continuous outcome variables: dependence of the magnitude of association and statistical power on the cutpoint. Epidemiology 3:434-440

30. Mattocks C, Leary S, Ness A et al (2007) Intra-individual variation of objectively measured physical activity in children. Med Sci Sports Exerc 39:622-629

31. Wong MY, Day NE, Wareham NJ (1999) Measurement error in epidemiology: the design of validation studies II: bivariate situation. Stat Med 18:2831-2845

32. Ekelund U, Franks PW, Wareham NJ, Åman J (2004) Oxygen uptakes adjusted for body composition in normal-weight and obese adolescents. Obes Res 12:513-520

33. Ekelund U, Sardinha LB, Anderssen SA et al (2004) Associations between objectively assessed physical activity and indicators of body fatness in 9- to 10-year old European children: a population based study from four distinct regions in Europe (The European Youth Heart Study). Am J Clin Nutr 80:584-590

34. Brage S, Wedderkopp N, Ekelund U et al (2004) Features of the metabolic syndrome are associated with objectively measured physical activity and fitness in children. The European Youth Heart Study (EYHS). Diabetes Care 27:2141-2148

35. Boreham CAG, Twisk J, Murray L, Savage M, Strain JJ, Gordon C (2001) Fitness, fatness, and coronary heart disease risk in adolescents: the Northern Ireland Young Hearts Project. Med Sci Sports Exerc 33:270-274

36. Schmitz KH, Jacobs DR, Hong CP, Steinberger J, Moran A, Sinaiko AR (2002) Association of physical activity with insulin sensitivity in children. Int J Obes 26:1310-1316

37. Shaibi GQ, Cruz ML, Ball GB et al (2005) Cardiovascular fitness and the metabolic syndrome in overweight Latino youths. Med Sci Sports Exerc 37:922-928

38. Bouchard C, Rankinen T (2001) Individual differences in response to regular physical activity. Med Sci Sports Exerc 33 (Suppl):S446-S451

39. Turpeinen JP, Leppavouri J, Hienonen OJ et al (2006) Muscle fiber type I influences lipid oxidation during low-intensity exercise in moderately active middle-aged men. Scand J Med Sci Sports 16:134-140 